\section{Thrombolytic Treatment}

Unlike anticoagulant therapy, treatment with thrombolytic drugs is aimed at accelerating lysis of existing emboli. Streptokinase was first used for pulmonary embolism by $\mathrm{N}$. L. Browse and D. C. O. James ${ }^{27}$ in four patients diagnosed as having pulmonary embolism, rapid clinical improvement being obtained in all. In $1968 \mathrm{~J}$. Hirsh and his colleagues ${ }^{28}$ reported 18 patients treated with streptokinase in whom there was angiographic assessment of severity of embolism before and after a 24-28 hour period of treatment. These authors made several very important observations. Firstly, angiographic and haemodynamic improvement were most marked in the four patients who had a 24-hour infusion of streptokinase and who had recent embolism uncomplicated by previous cardiorespiratory disease. Improvement was less apparent in the 12 patients who had either associated cardiorespiratory disease or clinical evidence of recurrent embolism. Secondly, the definite angiographic improvement seen after streptokinase contrasted with the lack of such improvement seen in patients after treatment with heparin; one such patient was then given streptokinase, with obvious clinical and angiographic improvement. Similar findings were reported by G. A. H. Miller et al. ${ }^{29}$ who obtained considerable haemodynamic and angiographic improvement following streptokinase in four patients with acute massive and two with acute minor pulmonary embolism, but little improvement in three patients with recurrent embolism. In one patient with acute massive pulmonary embolism there was no angiographic improvement after six days of heparin, but complete resolution occurred 48 hours after starting streptokinase.

Though spontaneous resolution of massive pulmonary embolism treated with heparin is known to occur, ${ }^{12}{ }^{30}$ the rate of such resolution would appear to be significantlv slower than can be obtained with streptokinase. Thus Tow and Wagner found complete resolution, as judged by lung scans, occurred in only 1 of 11 patients with severe or massive embolism (at the 60th day), the earliest partial resolution being observed at the eighth day. ${ }^{31}$ Recently J. E. Dalen and his coworkers used pulmonary arteriograms to assess resolution rate in 15 patients treated with heparin or venous ligation, or both, and found only minimal resolution at seven days. ${ }^{32}$ Similar findings have also been reported, ${ }^{33}$ and our own experience indicates that, over a period of 3-7 days, little angiographic (though some haemodynamic) improvement occurred in a heparin-treated group but that resolution was almost complete in a group investigated after a 72-hour streptokinase infusion. ${ }^{34}$

An initial dose of 600,000 units of streptokinase is infused over the first half hour followed by 100,000 units/hour for 72 hours. Although some patients, by reason of a high antistreptokinase titre, will require a higher dose, this standard dosage will provide effective fibrinolysis and with few side-effects in all but a few of the population. ${ }^{35}$ In our experience 72 hours of this treatment is adequate for almost complete lysis of emboli and is probably accompanied by a lower incidence of complica- tions than would follow a more prolonged period of treatment. Patients who at any time deteriorate while on such treatment or who, having been resuscitated from the initial event, fail to maintain an adequate circulation would be candidates for pulmonary embolectomy. Such clinical deterioration, "as evidenced by intolerably low levels of mean arterial pressure despite pressor support," is probably today the major indication for pulmonary embolectomy on cardiopulmonary bypass. ${ }^{21}$

The use of urokinase in pulmonary embolism has, at present, been confined to the U.S.A., where several reports of its efficacy in lysing pulmonary emboli have appeared. ${ }^{36-39}$ Again, these reports have indicated that urokinase results in more rapid lysis than does heparin treatment ${ }^{37} 38$ and that lysis is most rapid when there is no complicating cardiorespiratory disease. ${ }^{39}$ Many of the patients successfully treated with urokinase would previously have been considered candidates for embolectomy.

\section{Indications for Embolectomy}

While it may be accepted that thrombolytic therapy hastens resolution of emboli it has yet to be shown that this reduces the mortality. Certainly a low or zero ${ }^{34}{ }^{37}$ mortality has been reported for such treatment, and probably some critically ill patients will die unless there is rapid lysis and haemodynamic improvement; however, patients being treated in this way presumably represent those who, having survived long enough for investigation and treatment have a better prognosis than that overall for patients with massive pulmonary embolism. Even a controlled trial of treatment may not show a reduced mortality, since probably patients who deteriorate on medical treatment will be submitted to pulmonary embolectomy. While such deterioration is the main indication for embolectomy there is one other group for whom embolectomy may be indicated-namely, patients in whom there are contraindications to thrombolytic therapy. Such contraindications may apply equally to thrombolytic or anticoagulant therapy and are necessarily ill defined. They include a known bleeding diathesis, actively bleeding gastro-intestinal and genito-urinary lesions, severe renal or hepatic disease, severe systemic hypertension, pregnancy and recent surgery where bleeding would constitute a serious risk. Complications are almost solely due to bleeding from surgical wounds, venepuncture sites, etc., though febrile reactions can occur unless steroid cover is given.

The incidence of incomplete resolution with pulmonary hypertension as a late result of a single massive embolus is thought to be low but is unknown. Equally unknown is whether thrombolytic therapy, at the time of the initial embolus, will reduce the incidence of this serious complication. D. Phear found that 19 of 68 patients surviving pulmonary embolism had moderate to severe exertional dyspnoea and three of these patients died. ${ }^{40}$ However, many of his patients may have had recurrent emboli-a condition recognized as predisposing to pulmonary hypertension. It is to prevent this serious complication of deep venous thrombosis that patients should be maintained on oral anticoagulants unless the factors predisposing to venous thrombosis no longer exist.

\title{
Surgical Management of Massive Pulmonary Embolism
}

\section{PANETH, ${ }^{*}$ F.R.C.S.}

The operative removal of massive pulmonary emboli was first advocated by F. Trendelenburg in $1908,{ }^{41}$ who showed experimentally that the principles underlying his operation were correct. He never himself, however, had a successful case to report, and it was his pupil $M$. Kirschner who recorded the first successful case treated by this technique in $1924 .{ }^{42}$ The

* Consultant cardiothoracic surgeon, Brompton Hospital, London S.W.3. operative mortality remained exceedingly high, and it was only the development of cardiopulmonary bypass and the availability of pre-packed, sterile, disposable oxygenators that altered the picture of the treatment of this frequently lethal condition. Up to 1961 there were only sporadic reports of successful interventions, but then E. H. Sharpe performed the first operation involving the use of cardiopulmonary bypass. ${ }^{43}$ Since then there have been numerous reports of 
other successes using this technique and this has led to a revival of interest in pulmonary embolism. At the Brompton Hospital the first patient was operated on using cardiopulmonary bypass in 1963, and the surgical series now totals over 34 cases.

In parallel with the improved and more refined surgical technique now available, newer pharmacological thrombolytic agents have been developed and are presently on trial. Hence the indications for operation for massive pulmonary embolism have narrowed considerably. Whereas before the presence of a life-threatening obstruction to emptying of the right ventricle was in itself an indication for removal, it is now believed that thrombolytic therapy should be the first line of attack, provided that:

(1) The blood pressure can be stabilized and does not require repeated and/or increasing doses of vasopressors to maintain it.

(2) There is no evidence of recurrent massive embolism.

(3) There is no history of a bleeding diathesis, peptic ulceration, an operation, or trauma within the preceding thrce days of the major embolic episode.

Under these circumstances it is not safe to use streptokinase.

(4) Clinically the patient seems unlikely to survive another 8-12 hours from the start of thrombolytic therapy.

Streptokinase requires about this period of time before any improvement is observed. Operation is also indicated if the patient shows evidence of continued deterioration during the course of thrombolytic therapy.

\section{Anaesthesia}

One of the most important aspects of the surgical management of these cases is the induction of anaesthesia. When a modified Trendelenburg operation has to be performed, as described below, endotracheal intubation will already have been carried out, so that no other anaesthetic induction is necessary. When operation with cardiopulmonary bypass is to be performed it is important that the surgical team and bypass apparatus should be ready in the theatre before the start of the anaesthetic. The administration of any anaesthetic agent will counteract the intense peripheral vasomotor tone, which maintains the systemic blood pressure in these patients. Since the cardiac output is severely limited and fixed at a low level by the obstruction in the pulmonary artery, a fall in blood pressure followed by bradycardia and arrest will occur if the peripheral resistance is allowed to fall. Hence the administration of any anaesthetic agent must be preceded by the intravenous injection of a powerful peripheral vasoconstrictor, such as metaraminol, and repeated doses of this should be given if the blood pressure (which should be displayed on an oscilloscope from an indwelling arterial cannula) shows any signs of falling. The proper appreciation of the precarious cardiovascular state of these patients-whose appearance may be very deceptively good-has led to a considerable improvement in the operative results. In the properly conducted case the operative mortality should be about $4-6^{\prime \prime} \%$ in the group operated on with cardiopulmonary bypass.

\section{Operation}

The original Trendelenburg procedure involves, in essence, a period of outflow occlusion-by placing a clamp across the pulmonary trunk and the aorta through the transverse sinusand then opening the pulmonary trunk distal to this clamp and sucking out any thrombus. Inflow, rather than outflow, stasis is preferable. The modified Trendelenburg operation applies to those patients in whom external cardiac massage has to be continued up to the time of the removal of the embolus, since the obstruction is total and the right ventricle is completely ineffective from paralysis. In these cases there is no time to transfer the patient to a centre with bypass facilities available for embolectomy.

The heart is exposed by a vertical median sternotomy, opening the pericardium longitudinally. If the edges of the pericardium are stitched to the wound, or held out, then an excellent exposure of the pulmonary trunk and caval terminations is obtained. Non-crushing clamps are placed on the inferior vena cava and superior vena cava, in that order, and the heart is massaged empty. The pulmonary trunk is opened over a side clamp and as much thrombus as possible is sucked out from each branch in turn over a period of $1 \frac{1}{2}-2$ minutes, while the circulation is virtually arrested.

The circulation is then restored by clamping the incision in the pulmonary trunk and releasing the superior vena cava, thus allowing the heart to fill slowly, and only then gradually releasing the inferior vena cava so as not to overfill the heart while it is regaining its function and tone. If further emboli are to be extracted, the whole process can be repeated after about 15 minutes-allowing some cerebral, coronary, and renal perfusion to take place in the meantime-and even assisting the feeble heart action with massage if necessary. By this repeated extraction of thrombus, the pulmonary circuit may eventually be sufficiently cleared to allow the heart to maintain an output unaided.

Obviously this operation carries a very high risk. It has to be performed, firstly, on a presumptive diagnosis, and, secondly, on patients who would otherwise certainly die. The technique, however has been successfully used by Clarke in Birmingham (personal communication) and by $\mathrm{K}$. Vossschulte et al." and A. Encke et al. ${ }^{15}$ in Germany.

\section{Cardiopulmonary Bypass}

Under any other circumstances embolectomy is best performed using cardiopulmonary bypass. This technique offers the surgeon the best possible operating conditions, while at the same time correcting all the physiological derangements resul' ing from the obstruction.

The exposure is by the same vertical median sternotomy. Cannulae are inserted into the root of the aorta and via the appendage into the right atrium. By connecting these to the arterial and venous limbs of the bypass circuit the heart and lungs are totally circumvented; the right ventricle, moreover, is relieved of its overload; the aorta is perfused with fully oxygenated blood at a physiological minute volume and thereby the cerebral, coronary, and renal perfusions are restored to normal. In the past causes of death have been due to cerebral, renal, and coronary anoxia. The large oxygen debt, which is a feature of the severe case, is corrected. While physiological normality is being restored by the extracorporeal circuit, the pulmonary trunk is opened longitudinally and each pulmonary arterial tree is carefully, slowly, and scrupulously cleared of all visible emboli.

Finally, the lungs are inflated repeatedly by the anaesthetist so as to force left atrial blood back into the pulmonary arteries. This is an excellent method of pulmonary massage and more gentle and physiological than the technique advocated by D. A. Coolcy et al. ${ }^{16}$ of compressing and squeezing the lungs manually. After both sides have been shown to be clear by the presence of red blood filling each side in turn, the incision in the pulmonary trunk is closed and the heart is allowed to take over the circulation. There may still then be evidence of right ventricular failure and partial paralysis, requiring a further period of bypass support. Isoprenaline in small quantities may be administered to improve the performance of the heart. After routine decannulation, the pericardium is closed loosely and the sternum approximated with rubb:r tube drains placed in the pericardial sac.

In the properly conducted case the postoperative course is smooth and most gratifying. It is important that patients after operation are placed on anticoagulants for a minimum period of 3-6 months. In the presence of overt signs of thrombosis and stasis in the peripheral veins, however, anticoagulants should be continued until all the clinical signs of deep venous thrombosis and pelvic vein thrombosis have subsided. In the surgical series at the Brompton Hospital there has only been one case of subsequent pulmonary embolism following operation and in this case anticoagulants were not properly maintained in the postoperative period. In this series it has not been necessary to interfere in any way with the systemic venous system in the pelvis or legs. 